\title{
APLICACIONES INFORMÁTICAS DIRIGIDAS A JÓVENES Y ADULTOS CON DISCAPACIDAD INTELECTUAL PARA EL DESARROLLO DE LAS ÁREAS DE APOYO
}

\section{Computer-based applications aimed at youth and adults with intellectual disability for the development of support areas}

\author{
María Esther BAÑOS-GARCíA \\ Avda. Cantabria, s/n. 09006 Burgos \\ ebanyos@ubu.es \\ Fernando LeZcano-BARbero \\ Universidad de Burgos. Facultad de Educación \\ Esteban García-Maté \\ Universidad de Burgos. Escuela Politécnica Superior \\ Raquel CASADO-MuÑOZ \\ Universidad de Burgos. Facultad de Educación
} Universidad de Burgos. Escuela Politécnica Superior. Campus Rio Vena

Recepción: 26 de julio de 2016

Aceptación definitiva: 19 de abril de 2017

Resumen: Podemos decir que el uso de las Tecnologías de la Información y Comunicación está generalizado en todos los ámbitos, incluido en la educación. En el caso concreto de los jóvenes y adultos con discapacidad intelectual, parece obvio que las aplicaciones informáticas deben servir para mejorar los apoyos a analizar en las distintas áreas funcionales. Para este artículo se han localizado 56 aplicaciones informáticas, en inglés y/o español, a través del estudio de revisiones, artículos científicos y páginas web. 
Posteriormente, se ha evaluado su relación con las áreas de apoyo, identificando el vínculo de cada aplicación informática con cada una de las nueve áreas. Los resultados obtenidos corroboran que la brecha digital sigue aumentando y, en algunos casos, se mantiene un tratamiento de las actividades propuestas que promociona la imagen infantil del colectivo. Encontramos un escaso conocimiento de las áreas de apoyo entre los desarrolladores y un desequilibrio en las aplicaciones, dado que algunas áreas cuentan con una gran cobertura mientras otras tienen una presencia residual. Se recomienda la promoción de las áreas entre los desarrolladores informáticos y más formación de educadores y de jóvenes y adultos con discapacidad intelectual, para un empleo más productivo de las TIC.

Palabras clave: discapacidad intelectual; jóvenes y adultos; Tecnologías de la Información y Comunicación (TIC); áreas de apoyo; brecha digital; educación.

Abstract: The use of Information and Communication Technologies is widely spread in all fields, including education. In the case of young people and adults with intellectual disabilities, it seems obvious that applications should serve to improve those supports to provide in the different functional areas. In this manuscript, 56 computer applications, in English and/or in Spanish, have been found by means of the search and study of reviews, scientific articles and web pages. Then, its connection with the support areas was evaluated by identifying the link for each computer application with each of the nine support areas. The findings confirm that the digital divide continues to increase and, in some cases, the treatment of the proposed activities promoting a childlike picture of this group is maintained. We found a low level of awareness of the support areas among developers and an imbalance in the applications, since some areas have great coverage while others have a residual presence. Promoting the consideration of the support areas among developers and further training for both educators and youth and adults with intellectual disabilities in order to reach a more productive use of ICT is recommended.

KEYwords: intellectual disability; youths and adults; Information and Communication Technologies (ICT); support areas; digital divide; education.

\section{$\tau$}

a Convención Internacional sobre los Derechos de las Personas con DisCAPACIDAD de 2006, en su artículo 19, titulado Derecho a vivir de forma independiente y a ser incluido en la comunidad, reconoce "[...] el derecho en igualdad de condiciones de todas las personas con discapacidad a vivir en la comunidad, con opciones iguales a las de los demás, para lo que se deben adoptar medidas efectivas y pertinentes [...]". A pesar de tener reconocido este derecho, entre otros muchos, con demasiada frecuencia las personas con discapacidad intelectual (DI) deben enfrentarse a numerosos inconvenientes a la hora de independizarse (Ramdoss et al., 2012). Entre estos problemas destacamos las dificultades que muchas veces encuentran al adquirir las habilidades necesarias para el desenvolvimiento diario (Gutiérrez y Martorell, 2011; Hutcherson, Langone, Ayres y Clees, 2004; Ramdoss et al., 2012; Savidis, Grammenos y Stephanidis, 2007; Stendal, 2012) y las dificultades 
para la provisión de los apoyos necesarios en su consecución (AAIDD, 2010; Pablo y Rodríguez, 2010; Schalock y Verdugo, 2003; Verdugo y Gutiérrez, 2009).

Para dar una respuesta adaptada a las necesidades de cada una de las personas, es fundamental la definición del perfil de apoyo; identificar el tipo y la intensidad de apoyos necesarios y las personas responsables de proporcionarlos en cada una de las nueve áreas (Verdugo y Schalock, 2010): 1) Desarrollo humano; 2) Enseñanza y educación; 3) Vida en el hogar; 4) Vida en la comunidad; 5) Empleo; 6) Salud y seguridad; 7) Conductual; 8) Social; y 9) Protección y defensa.

La necesaria individualización, tanto en la definición del perfil como en su posterior desarrollo, hace que tome gran interés el hecho de que la persona responsable del seguimiento cuente con el mayor número de posibilidades para su puesta en marcha. Tradicionalmente, los apoyos han sido prestados por personas: profesionales, familiares, voluntarios... Estas personas contaban con diferentes recursos, pero el desarrollo de las Tecnologías de la Información y la Comunicación (TIC) ha conseguido que, también en el ámbito de la discapacidad, los programas informáticos y las aplicaciones se conviertan en un elemento que se debe tener en cuenta.

Aunque el empleo de ordenadores, el acceso a Internet y, en general, el uso de las TIC están abriendo nuevas y múltiples posibilidades en educación, salud, ocio o vida social (Aguilar, 2004; Buedo y Molina, 2007; Gutiérrez y Martorell, 2011; Suárez, 2007), aquellos colectivos que, o bien no posean las habilidades necesarias en el manejo de TIC, o bien no tengan acceso a ellas, pueden quedar excluidos de esta sociedad de la información (Casado-Muñoz, Lezcano y Rodríguez-Conde, 2015; Gutiérrez y Martorell, 2011; Maeda et al., 2009; McKenzie, 2007 citado por Abbot et al., 2011), ampliando cada vez más la llamada brecha digital (OECD, 2001) y, con ella, el aislamiento y las desventajas económicas (Dekelver y Boeck, 2010) o de otra índole.

Estas diferencias en cuanto al acceso, manejo y empleo de las TIC guardan una importante relación con la normalización didáctica (Casado-Muñoz, 2002), por la que es de esperar que las personas con DI trabajen con materiales educativos adaptados a sus características, en situación equiparable a sus compañeros sin discapacidad (Savidis et al., 2007).

Debemos atender a las nuevas oportunidades que las TIC abren a las personas con discapacidad (Davies, Stock y Wehmeyer, 2001) y aprovechar las prometedoras perspectivas que les pueden capacitar al mismo nivel que los demás (Stendal, 2012). Como afirman Leahy y Dolan (2010), la exclusión social se puede reducir o eliminar si sabemos usar adecuadamente estas tecnologías.

La formación y entrenamiento de destrezas de la vida real en personas con DI supone un reto difícil y exigente que puede ser alcanzado con el empleo de instrumentos de software de propósito específico (Davies, Stock y Wehmeyer, 2001; Savidis, Grammenos y Stephanidis, 2007). Wells, Clark y Sarno (2014) van más allá y exponen la necesidad de desarrollar, a su vez, propuestas de trabajo basadas en actividades multimedia de carácter interactivo (CBIM: Computer-based Interventions Multimedia) que incluyan un amplio abanico de experiencias y permitan a las personas con DI interactuar con el ordenador en los diferentes contextos donde desarrollan sus actividades habituales (entornos de formación, contextos laborales, espacios de socialización...). 
Actualmente, el acceso de la población general a programas o aplicaciones informáticas (AI), ya sean de acceso libre o de pago, en la Red o en soportes portátiles, podríamos decir que es casi imposible de calcular, dado que diariamente nos encontramos con nuevos programas, constantes actualizaciones o nuevas adaptaciones. Aunque muchas de ellas pueden ser utilizadas por personas con DI para adquirir, entrenar, mejorar distintas habilidades, o simplemente divertirse, esta situación, para nuestro colectivo, es notablemente más restrictiva.

También podemos buscar aplicaciones construidas o seleccionadas específicamente para las personas con DI. En este caso, la realidad que se presenta se podría calificar de compleja o incluso caótica. Numerosos factores han influido en esta situación: el reducido tamaño de mercado que se genera; la frecuente falta de coincidencia entre el usuario final y quien adquiere/solicita la aplicación (Hersh, 2010); factores financieros y comerciales que, en muchas ocasiones, han hecho prevalecer aquellos proyectos mejor financiados, independientemente de sus valores o virtudes.

Tampoco entre los investigadores encontramos objetivos y modelos claros, surgiendo diferencias de opiniones. Por un lado, están aquellos partidarios de crear aplicaciones individualizadas y adaptadas a colectivos e individuos concretos (Savidis et al., 2007), mientras que otros prefieren programas más generalistas (iguales para todo tipo de usuarios), aunque siempre se cuente con la posibilidad de disponer de distintas adaptaciones, según distintos tipos de discapacidad (Sánchez, 2002).

Esta diversidad también se ve reflejada en la literatura científica sobre recopilaciones de aplicaciones tecnológicas para personas con DI (Abbott, 2007; Abbott et al., 2011; Sánchez, 2002), donde comprobamos la enorme disparidad de criterios que existen a la hora de catalogarlas. Esa disparidad se encuentra también en el campo general de la tecnología, donde distintas clasificaciones incluyen criterios como: objetivos y colectivos receptores (Marqués, 2010), modelos pedagógicos de apoyo y tipo de contenidos (García-Valcárcel, 2009; Marqués, 2010), características cognitivas del usuario final (Gros, 2003), comportamiento didáctico de la aplicación (Bull, 2009; Hinostroza et al., 1997; Marqués, 2010), elementos técnicos de diseño (García-Valcárcel, 2009), etc.

La terminología concreta con la que cada autor/investigador/diseñador/usuario/ comercial... designa los diferentes recursos tecnológicos también es diferente. Esto puede provocar cierta discordancia al comparar las distintas catalogaciones y su empleo posterior (Hersh, 2010).

Finalmente, es importante señalar la obsolescencia, cada vez más marcada, de buena parte de los recursos actualmente disponibles. Esta situación ya fue señalada en el Informe del grupo de trabajo para el Tercer Congreso Internacional de EducaRed (2005), y no ha mejorado con el paso de los años, en el ámbito del presente trabajo.

Ante esta situación (disparidad de criterios de construcción, de denominación, de organización...) nos planteamos no volver a crear un nuevo catálogo y unos nuevos criterios de organización, sino identificar aquellas aplicaciones más adecuadas para el desarrollo de la autonomía de las personas con DI y disponerlas en relación con las áreas de apoyo funcional, habitualmente utilizadas en la evaluación de los perfiles de necesidades de apoyo. 
En suma, en esta investigación, nos planteamos conseguir los siguientes objetivos:

- Identificar las aplicaciones informáticas que se encuentran accesibles y operativas para personas con DI, familias y/o formadores.

- Evaluar la relación de las aplicaciones con las áreas de apoyo definidas por la AAIDD.

\section{Método}

\subsection{Definición de la población diana}

Dada la compleja situación de las aplicaciones informáticas para el colectivo, descrita anteriormente, se decidió identificar solo aquellas dirigidas a jóvenes y adultos. La búsqueda de aplicaciones para niños se manifiesta extremadamente más compleja si cabe, dado que existe un número muy elevado de aplicaciones vinculadas a distintas asignaturas de cada uno de los niveles educativos.

\subsection{Selección del idioma}

Para delimitar correctamente las búsquedas, se seleccionan los idiomas inglés y español, dado que son los que cuentan con una mayor difusión.

\subsection{Delimitación del periodo temporal}

El periodo cubierto para la revisión fue desde 1996 hasta 2016. No obstante, se ha profundizado en el estudio de los textos y aplicaciones presentados en la última década, por la ya referida obsolescencia y falta de actualización de este tipo de herramientas.

El sistema de búsquedas ha abarcado diferentes fuentes:

- Documentación científica. Se realizaron búsquedas sistemáticas de artículos de revistas; comunicaciones recogidas en actas de Congresos, seminarios y jornadas, y libros, en bases de datos (Web of Science, Scopus, ERIC, PubSych) y, además, se siguieron otras rutas secundarias de búsqueda como Medline, Dialnet y Google Scholar.

- Internet. Para ello hemos trabajado con numerosas páginas de distintas asociaciones que agrupan al colectivo de personas con DI, en las que a veces se relacionan recursos de apoyo o direcciones donde recurrir; organismos e instituciones oficiales, de España y de otros países, que trabajan sobre políticas relacionadas con el colectivo, su educación y su inclusión; editoriales y compañías que diseñan y distribuyen este tipo de recursos; centros de investigación y organizaciones sin ánimo de lucro, que incluyen entre sus objetivos la promoción de la inclusión de personas con DI por medio del uso de las TIC. 
Las palabras clave utilizadas en los distintos motores de búsqueda han sido, en español, con diferentes combinaciones: "multimedia”, “interactiv*” "aplicación”, "software”, "realidad virtual”, "discapacidad intelectual”, "dificultad* de aprendizaje”, "necesidad" especial* educación”, "revisión”, "recopilación”; y en inglés, evidentemente también con diferentes combinaciones: "multimedia”, "interactiv*”, "software”, "virtual reality”, “intellectual disability”, "learning difficult*”, "review”, "revision”.

En síntesis, los recursos posibles a incluir debían ser: a) aplicaciones multimedia dirigidas a la formación o entrenamiento de cualquier destreza, en personas con discapacidad intelectual o con dificultades de aprendizaje, b) dirigida a jóvenes y adultos y c) diseñadas en español y/o en inglés.

En el proceso de búsqueda de aplicaciones informáticas se encontraron, inicialmente, 380 posibles propuestas. De todas ellas, 188 se recogían en artículos científicos y 192 en páginas web.

\section{A) Búsqueda en bases de datos}

Se localizaron nueve revisiones (review) en otros tantos artículos. Con cinco de ellas se realizó un estudio exhaustivo identificando el conjunto de aplicaciones recogidas y su localización (Tabla 1). Así mismo, se contrastan las aplicaciones repetidas. Las otras cuatro se descartaron por no ajustarse a los criterios indicados ya que tratan recursos como: a) empleo exclusivo de mundos virtuales (VW) que requieren mucha tecnología y muy compleja; b) comparativas entre VW, instrucciones trabajadas en aulas e instrucciones trabajadas en los propios ambientes reales (cocina, calle, etc.); c) sistemas no multimedia (basados solo en disposición y visualización de imágenes, o solo vídeos o audios, sin ningún tipo de interactividad); y d) tecnologías asistidas dirigidas totalmente a personas con discapacidades funcionales o del desarrollo.

\begin{tabular}{|l|c|c|l|l|}
\hline \multicolumn{5}{|c|}{$\begin{array}{l}\text { TABLA 1. Revisiones sobre aplicaciones multimedia dirigidas a personas } \\
\text { con discapacidad intelectual o con dificultades de aprendizaje }\end{array}$} \\
\hline REFERENCIA & ${\text { N. }{ }^{\circ} \text { ESTUDIOS }}^{\text {EDAD }}$ & \multicolumn{1}{|c|}{ TECNOLOGÍA } & RESULTADO \\
\hline $\begin{array}{l}\text { Cullen y Albert- } \\
\text { Morgan (2015) }\end{array}$ & 36 & $12-48$ & Technology mediated Self-Prompting & $\begin{array}{l}\text { Positivos 25 } \\
\text { A estudiar 9 } \\
\text { Negativos 2 }\end{array}$ \\
\hline $\begin{array}{l}\text { Ramdoss et al. } \\
\text { (2012) }\end{array}$ & 11 & $9-58$ & $\begin{array}{l}\text { Software “I can! Daily Living and Community } \\
\text { Skills"_2 } \\
\text { "Project Shop"_3 } \\
\text { "Hyperstudio"_4 } \\
\text { "ATM Sim"_1 } \\
\text { "Power Point Windows Movie Maker"_1 8 }\end{array}$ & $\begin{array}{l}\text { A estudiar 3 } \\
\text { Negativos 0 }\end{array}$ \\
\hline Mechling (2008) & 22 estudios & revisiones & $\begin{array}{l}\text { Picture-based system 7 } \\
\text { Palmtop PC_3 } \\
\text { Auditory Systems_5 } \\
\text { Video-based systems 7 }\end{array}$ & $\begin{array}{l}\text { Positivos 19 } \\
\text { A estudiar 3 } \\
\text { Negativos 0 }\end{array}$ \\
\hline
\end{tabular}

(C) Ediciones Universidad de Salamanca / CC BY-NC-ND

Siglo Cero, vol. 48 (2), n. ${ }^{\circ}$ 262, 2017, abril-junio, pp. 7-24 
TABLA 1. Revisiones sobre aplicaciones multimedia dirigidas a personas con discapacidad intelectual o con dificultades de aprendizaje

\begin{tabular}{|c|c|c|c|c|}
\hline REFERENCIA & N. ${ }^{\circ}$ ESTUdios & EDAD & Tecnología & Resultado \\
\hline $\begin{array}{l}\text { Maccini, Calvin- } \\
\text { Gagnon y Hughes } \\
(2002)\end{array}$ & 10 & $12-19$ & $\begin{array}{l}\text { Video disc_2; Word procesor lecture_5; Audio- } \\
\text { based__1; Class maps_1; Class worksheet_2; } \\
\text { Computer study guide_4; Hypermedia study } \\
\text { guide_2; Computerized map tutorial_1 }\end{array}$ & $\begin{array}{l}\text { Positivos } 7 \\
\text { A estudiar } 3 \\
\text { Negativos } 0\end{array}$ \\
\hline Stendal (2012) & 54 & $\begin{array}{c}\text { No } \\
\text { indicado }\end{array}$ & $\begin{array}{l}\text { Web-based_31; } \\
\text { e-learning tool_6; Assistive Technology } 5 \\
\text { Other ICT_9; Custom designed VW_8 } \\
\text { Open-access Multi-user VW_4 }\end{array}$ & Sin datos \\
\hline
\end{tabular}

De los 179 estudios restantes recuperados de las bases de datos, se estudiaron en profundidad 24 artículos, el resto fueron descartados por tratar de aplicaciones que incumplían nuestros criterios. De entre los seleccionados, sólo 3 reseñaron claramente el desarrollo y estudio de 14 aplicaciones multimedia interactivas, actualmente accesibles para soportes portables como ordenadores, tabletas, móviles... Debemos señalar que, aunque 14 de estos artículos también informan de la evaluación de algún tipo de aplicación multimedia, no se han incluido en la colección por varias razones: a) son aplicaciones dirigidas inicialmente a niños, aunque luego se haya estudiado su efecto también en jóvenes e incluso adultos con DI, y desarrollan destrezas específicas del contexto escolar, escasamente generalizables para el desenvolvimiento independiente diario; o b) son presentaciones Power Point sin ningún tipo de interactividad.

\section{B) Búsqueda en páginas web}

En el caso de las páginas web, se encontraron 192, de las cuales 65 corresponden a páginas comerciales; 52 a instituciones gubernamentales, fundaciones o centros de investigación; 35 a asociaciones u organizaciones no gubernamentales; 4 de canales de televisión públicos que dedican programas específicos; 8 de particulares; 7 blogs y wikis, y 21 páginas que se incluyeron en el apartado de "otros" (proyectos educativos de colegios o páginas donde no encontramos referencias concretas).

Todas ellas se revisan en profundidad dando como resultado: 19 webs que indican aplicaciones multimedia que se ajustan a nuestros criterios. De ellas 14 corresponden a páginas de distribuidores y desarrolladores con metas comerciales, 1 a una fundación y 4 a instituciones oficiales y asociaciones relacionadas con el colectivo. Como es de esperar, algunos estudios están citados en dos o más de las revisiones incluidas, o las distintas páginas web hacen referencia a los mismos recursos educativos. El resultado final son las 56 aplicaciones que se recogen en la Tabla 2. En esta tabla se indican el nombre y la web en la que se pueden encontrar. 
APLICACIONES INFORMÁTICAS DIRIGIDAS A JÓVENES Y ADULTOS CON DISCAPACIDAD INTELECTUAL

PARA EL DESARROLLO DE LAS ÁREAS DE APOYO

M. E. BAÑOS-GARCÍA, F. LEZCANO-BARBERO, E. GARCÍA-MATÉ Y R. CASADO-MUÑOZ

\section{TABLA 2. Listado de las 56 aplicaciones localizadas y estudiadas}

\begin{tabular}{|c|c|c|}
\hline N. ${ }^{\circ}$ & Título. ReFerencia & ACCESO \\
\hline 1 & El arca de los pensamientos & http://bit.ly/1RiODx7 \\
\hline 2 & Auditory discrimination and processing: Nouns and sounds & http://bit.ly/1mTiMZ7 \\
\hline 3 & Auditory discrimination and processing: The following direction series & http://bit.ly/1mTiMZ7 \\
\hline 4 & Categorization skills: First categories and twenty categories & http://bit.ly/1mTiMZ7 \\
\hline 5 & Cheese factory & http://bit.ly/1Qcotxd \\
\hline 6 & Chooselt! ready-made numeracy series: Money & $\begin{array}{l}\text { http://bit.ly/1RZsRRF } \\
\text { http://bit.ly/1T207sB }\end{array}$ \\
\hline 7 & CITI (Orange Foundation) & http://bit.ly/1XIDdW1 \\
\hline 8 & Community Construction Kit & http://bit.ly/12Kq8oB \\
\hline 9 & Community success & $\begin{array}{l}\text { http://bit.ly/1RiPk9H, } \\
\text { http://bit.ly/1QVRWYp }\end{array}$ \\
\hline 10 & Diorama designer & http://bit.ly/1Q6fJoT \\
\hline 11 & Disco & http://bit.ly/1QxDETF \\
\hline 12 & Dollars and Cents software & http://bit.ly/20QPQ1j \\
\hline 13 & Focus on feelings & http://bit.ly/1Qu4ndy \\
\hline 14 & Functional language: The language activities of daily living series & http://bit.ly/1mTiMZ7 \\
\hline 15 & Grocery Word and shopping list generator & http://bit.ly/1QVUc1V \\
\hline 16 & Habitúate [Get used to] & http://bit.ly/1L6vAGY \\
\hline 17 & Lifeskill: 24 hour a day & $\begin{array}{l}\text { http://bit.ly/1TAPXi6 } \\
\text { http://bit.ly/1T207sB }\end{array}$ \\
\hline 18 & Live safe: preventing HIV/AIDS for women with ID & http://bit.ly/1VzSJSL \\
\hline 19 & Live smart live safe: preventing HIV/AIDS for men with ID & http://bit.ly/1VzSJSL \\
\hline 20 & Live smart live safe: Emergency preparedness & http://bit.ly/1VzSJSL \\
\hline 21 & Live smart live safe: preventing respiratory illness & http://bit.ly/1VzSJSL \\
\hline 22 & Lucas y el caso del cuadro robado & http://bit.ly/1XIDdW1 \\
\hline 23 & Memobile & $\begin{array}{l}\text { http://bit.ly/1oYhLR8 } \\
\text { Para móvil y PC }\end{array}$ \\
\hline 24 & Mind Reading: the interactive guide to emotions 1.3 & http://bit.ly/1Q6j5Iv \\
\hline 25 & Moneytalks! & http://bit.ly/1L6tqHs \\
\hline 26 & My appearance & http://bit.ly/1oYhLR8 \\
\hline 27 & $\begin{array}{l}\text { My community: achieving everyday social success through real life video } \\
\text { scenarios }\end{array}$ & $\begin{array}{l}\text { http://bit.ly/1oYhX2H } \\
\text { http://bit.ly/1XIDMiF }\end{array}$ \\
\hline 28 & Neighborhood mapmachine & http://bit.ly/12Kq8oB \\
\hline 29 & Out and about 1 plus - The living community & http://bit.ly/21lyGLq \\
\hline 30 & Out and about 2 plus - Around the home & $\begin{array}{l}\text { http://bit.ly/1UixYwo } \\
\text { http://bit.ly/1oDDUnW }\end{array}$ \\
\hline 31 & Out and about 3 plus - Gadgets at home & $\begin{array}{l}\text { http://bit.ly/1OqAB85 } \\
\text { http://bit.ly/1oDDUnW }\end{array}$ \\
\hline
\end{tabular}


APLICACIONES INFORMÁTICAS DIRIGIDAS A JÓVENES Y ADULTOS CON DISCAPACIDAD INTELECTUAL

PARA EL DESARROLLO DE LAS ÁREAS DE APOYO

M. E. BAÑOS-GARCÍA, F. LEZCANO-BARBERO, E. GARCÍA-MATÉ Y R. CASADO-MUÑOZ

\section{TABLA 2. Listado de las 56 aplicaciones localizadas y estudiadas}

\begin{tabular}{|c|c|c|}
\hline N. ${ }^{\circ}$ & Título. Referencia & ACCESO \\
\hline 32 & Out and about 4 - Money and finance & $\begin{array}{l}\text { http://bit.ly/1QVUZQk } \\
\text { http://bit.ly/1oDDUnW }\end{array}$ \\
\hline 33 & Out and about - assistive technology: assessing needs & http://bit.ly/1oDDUnW \\
\hline 34 & Personal success & $\begin{array}{l}\text { http://bit.ly/1WEeIYU } \\
\text { http://bit.ly/1oDDUnW }\end{array}$ \\
\hline 35 & Pictogram room & http://bit.ly/1LJUgAR \\
\hline 36 & Picture cue dictionary & $\begin{array}{l}\text { http://bit.ly/1SNSFRf } \\
\text { http://bit.ly/1oDDUnW }\end{array}$ \\
\hline 37 & Read to learn software bundle & $\begin{array}{l}\text { http://bit.ly/1mTlXQz } \\
\text { http://bit.ly/1oDDUnW }\end{array}$ \\
\hline 38 & Shop'til you drop & http://bit.ly/1RiSPwQ \\
\hline 39 & Life Skills & $\begin{array}{l}\text { http://bit.ly/1RiPk9H } \\
\text { http://bit.ly/1oDDUnW }\end{array}$ \\
\hline 40 & Social skills at work & http://bit.ly/1oDDUnW \\
\hline 41 & Social skills on the job & $\begin{array}{l}\text { http://bit.ly/1RiPk9H } \\
\text { http://bit.ly/1oDDUnW }\end{array}$ \\
\hline 42 & Starting work (Isometric game) & http://bit.ly/1oYhLR8 \\
\hline 43 & Switchit! Hygiene extra & $\begin{array}{l}\text { http://bit.ly/1QGc0xA } \\
\text { http://bit.ly/1T207sB }\end{array}$ \\
\hline 44 & Switchit! Maker 2 older learners edition & $\begin{array}{l}\text { http://bit.ly/1OHrKPC } \\
\text { http://bit.ly/1T207sB }\end{array}$ \\
\hline 45 & Teaching Access ability & $\begin{array}{l}\text { http://bit.ly/1L6xTtC } \\
\text { http://bit.ly/1QcsTEi }\end{array}$ \\
\hline 46 & Timescales & $\begin{array}{l}\text { http://bit.ly/1Q6kNtk } \\
\text { http://bit.ly/1T207sB }\end{array}$ \\
\hline 47 & True/False and conveyor belt quiz: I Personal Hygiene & http://bit.ly/1oYhLR8 \\
\hline 48 & True/False and conveyor belt quiz: II Getting and keeping a job & http://bit.ly/1oYhLR8 \\
\hline 49 & True/False and conveyor belt quiz: III Stress at work & http://bit.ly/1oYhLR8 \\
\hline 50 & True/False and conveyor belt quiz: IV Anger management & http://bit.ly/1oYhLR8 \\
\hline 51 & Virtual supermarket & http://bit.ly/1oYhLR8 \\
\hline 52 & Vocabulary and concept development: The words \& concepts series & http://bit.ly/1ReKMia \\
\hline 53 & Vocabulary and concept development: The concentrate! Series & http://bit.ly/1LpJd4c \\
\hline 54 & Whose future is it? & $\begin{array}{l}\text { http://bit.ly/1TK75RA } \\
\text { http://bit.ly/1T207sB }\end{array}$ \\
\hline 55 & You are a social detective! & $\begin{array}{l}\text { http://bit.ly/1LCLO5A } \\
\text { http://bit.ly/1QxH8W7 }\end{array}$ \\
\hline 56 & Proyecto Aprender & http://bit.ly/1SLQNrw \\
\hline
\end{tabular}

(C) Ediciones Universidad de Salamanca / CC BY-NC-ND

Siglo Cero, vol. 48 (2), n. ${ }^{\circ}$ 262, 2017, abril-junio, pp. 7-24

$$
-15 \text { - }
$$




\section{Evaluación de las áreas funcionales}

Para realizar la evaluación de las aplicaciones encontradas y su relación con las áreas de apoyo funcional, se propone la revisión de las aplicaciones por dos equipos de trabajo compuestos, cada uno de ellos, por un ingeniero y un pedagogo, en ambos casos especialistas en personas con discapacidad intelectual.

Cada equipo debía revisar las distintas aplicaciones indicando si las mismas trabajan (Sí/No) cada una de las áreas de apoyo. En el caso de encontrar discrepancias se discutiría el criterio aplicado buscando un consenso.

Tras una valoración inicial aparecieron bastantes discrepancias entre ambos equipos. Por ello se realiza una especificación de tales áreas a partir de la descripción propuesta por la AAIDD $(2010,2012)$. El resultado de la subdivisión de las áreas de apoyo queda recogido en la Tabla 3.

TABLA 3. Subdivisión de cada una de las Áreas de Apoyo. A partir de AAIDD $(2010,2012)$

DeSARROLlO HUMANO
a) Habilidades prácticas: psicomotoras.
b) Habilidades sociales.
c) Habilidades conceptuales: comunicación y lenguaje,
procesos cognitivos básicos (percepción, atención,
memoria, lenguaje).

\begin{tabular}{|l|l}
\hline VIDA EN EL HOGAR & VIDA EN LA COMUNIDAD \\
\hline
\end{tabular}

a) Organización del espacio personal.

c) Limpieza (no higiene).

d) Tareas básicas de limpieza (barrer, limpiar, fregar...).

e) Cocinar.

f) Ocio en el hogar (TV, música, juegos...).

EMPLEO

a) Preparación del currículum.

b) Enfrentarse a una entrevista.

c) Conocimiento de las capacidades individuales.

d) Búsqueda y mantenimiento del puesto de trabajo. b) Compra (productos de limpieza, comida).

\section{ENSEÑANZA Y EDUCACIÓN}

a) Lectura, escritura.

b) Cálculo.

c) Toma de decisiones.

d) TIC para la educación.

VIDA EN LA COMUNIDAD

a) Uso de transportes públicos y elección de itinerarios

b) Uso de servicios públicos: hospitales, ambulatorios, bancos...

c) Participación social: Iglesia, fiestas, centros sociales...

d) Realización de compras: manejo de dinero, precio ajustado, prioridad de las necesidades.

SALUD Y SEGURIDAD

a) Servicios sanitarios: solicitar cita, sintomatología de las enfermedades, seguimiento de los tratamientos.

b) Servicios de emergencias: ambulancias, policía, bomberos...

c) Salud: alimentación saludable, ejercicio...

d) Educación vial, prevención del riesgo.

e) Seguridad en el hogar.

SOcial (diferenciar de vida en la comunidad)

CONDUCTUAL

a) Establecimiento de relaciones significativas.

a) Autonomía personal.

b) Mejora de la comunicación: intereses, preferencias.

c) Autodirección.

d) Autorregulación.

e) Cumplimiento de horarios. b) Mantenimiento de relaciones familiares, amistades y de pareja (juegos, fiestas con amigos...). 
APLICACIONES INFORMÁTICAS DIRIGIDAS A JÓVENES Y ADULTOS CON DISCAPACIDAD INTELECTUAL

PARA EL DESARROLLO DE LAS ÁREAS DE APOYO

M. E. BAÑOS-GARCÍA, F. LEZCANO-BARBERO, E. GARCÍA-MATÉ Y R. CASADO-MUÑOZ

TABLa 3. Subdivisión de cada una de las Áreas de Apoyo. A partir de AAIDD (2010, 2012) PROTECCIÓN Y DEFENSA

a) Administración: gestión de facturas, recibos, ahorro, tarjetas...

b) Servicios legales y protección pública: búsqueda y asesoramiento legal, sindicatos, seguros, oficina del consumidor...

c) Derechos y responsabilidades legales: normativa urbana, comportamiento cívico, respeto del turno...

d) Evitar riesgos: situaciones de explotación, abuso (físico, psíquico).

En esta reformulación, se incluye un apartado o subárea nueva que se estima de interés para el estudio: 'TIC para la educación', aunque es un apartado que no se contempla en ninguno de los documentos de la AAIDD (2010, 2012).

Con esta nueva propuesta, las discrepancias entre los equipos se redujeron de forma sustancial y en los pocos apartados en los que se encontraron juicios diferentes, se debatió hasta llegar al consenso, lo cual se consiguió fácilmente.

Se valoraron las 56 aplicaciones encontradas en relación con las subáreas y áreas funcionales, obteniendo los resultados que se recogen en la Tabla 4. Dada la extensión de la Tabla 4 se incluye como descargable en http://bit.ly/2oawkU7 e imprimible en A3.

\section{Análisis}

Encontramos un considerable desequilibrio en las aplicaciones que trabajan cada una de las áreas funcionales aunque, antes de avanzar, debemos realizar algunas matizaciones. El área de "Desarrollo humano" es la que cuenta con una mayor presencia, hasta un 70,85\% de los programas evaluados desarrollan alguna de sus subáreas. Pero esta elevada tasa debe ser analizada de forma crítica, dado que dos de las subáreas evaluadas (a/ aspectos psicomotrices y c/ procesos cognitivos básicos) se trabajan con todas las aplicaciones identificadas o, dicho de otra forma, cualquier aplicación informática, sea específica para el colectivo o más generalista, realizaría la misma función. Esto sucede con la aplicación número 45 (Teaching Access ability) que aborda sólo esta área y podría ser sustituida por cualquier otra aplicación, aunque no fuera específica para el colectivo, dado que, al menos, desarrollaría las mismas habilidades.

Las áreas Conductual (30,91\%) y Enseñanza y educación $(34,09 \%)$ son las que cuentan con mayor presencia entre las aplicaciones. Mientras en la primera de las áreas podemos observar que se trabajan muchas de las subáreas, entendemos que la puntuación media es representativa de esta realidad. Esto no sucede en el caso de Enseñanza y educación, pues la valoración media viene ampliamente distorsionada por la alta puntuación de la subárea (c) Toma de decisiones. Si realizamos una estimación de cambio eliminando la puntuación de esta subárea (c), encontramos que la puntuación media del área disminuiría hasta el 20\%, ubicándola en una posición notablemente inferior en relación con la actual.

En el resto de las áreas se trabaja solamente con un $20 \%$ de las aplicaciones o menos. Vida en la comunidad sólo se trabaja en un 13,18\% de las aplicaciones; Seguridad 
y Salud en un $11,63 \%$ de las mismas; Empleo en un 7,27\% de los casos, y Vida en el hogar en un 6,36\% de las aplicaciones evaluadas. Especialmente llamativo es el caso de Protección y defensa, que sólo se trabaja en un 1,8\% de las aplicaciones.

La división por subáreas nos permite visualizar con mayor claridad la realidad con la que nos encontramos, por ello no debemos dejar de comentar que en el conjunto de las áreas son varias las subáreas que no se contemplan.

Estudiando con mayor detalle, podemos ver que algunas subáreas cuentan también con escasa presencia en las aplicaciones analizadas. A continuación, se recogen aquellas subáreas que se trabajan en dos o menos aplicaciones, de las 56 analizadas:

- Preparación del currículum (Empleo).

- Enfrentarse a una entrevista (Empleo).

- Limpieza (no higiene) (Vida en el hogar).

- Cocinar (Vida en el hogar).

- Ocio en el hogar (TV, música, juegos...) (Vida en el hogar).

- Servicios legales y protección pública: búsqueda y asesoramiento legal, sindicatos, seguros, oficina del consumidor... (Protección y defensa).

- Derechos y responsabilidades legales: normativa urbana, comportamiento cívico, respeto del turno... (Protección y defensa).

- Evitar riesgos: situaciones de explotación, abuso (físico, psíquico) (Protección y defensa).

Por otro lado, a la hora de elegir una aplicación es importante, además de tener en cuenta el área específica que se desee trabajar, otras áreas que se aborden a la vez.

En la situación menos favorable, como ya hemos indicado, se encuentra la aplicación número 45 (Teaching Access ability), dado que sólo aborda un área, pudiéndose sustituir por cualquier otra aplicación, incluso no específica para el colectivo. En una situación similar hallamos las aplicaciones $4,13,15,35,44,46,47,48$ y 52, que trabajan solamente 2 áreas. Contando que, como hemos indicado, el área de Desarrollo humano se trabaja con todas las aplicaciones, estas sólo se deberían seleccionar cuando se desee trabajar el área en concreto que específicamente desarrollan.

Con mayor interés encontramos aquellas que se ocupan de un elevado número de áreas a la vez. Del conjunto estudiado, destacamos aquellas que trabajan 6 o 7 de las áreas:

\begin{tabular}{|l|l|}
\hline 6 áreas & $\begin{array}{l}\text { 26. My appearance. } \\
\text { 30. Out and about 2 plus - Around the home. } \\
\text { 37. Read to learn software bundle. } \\
\text { 39. Life Skills. } \\
\text { 51. Vocabulary and concept development: The words and concepts series. }\end{array}$ \\
\hline 7 áreas & $\begin{array}{l}\text { 25. Moneytalks! } \\
\text { 32. Out and about } 4 \text { - Money and finance. } \\
\text { 36. Picture cue dictionary. }\end{array}$ \\
\hline
\end{tabular}

Estudiando específicamente el caso de las aplicaciones que se pueden encontrar en español, sólo nos podemos referir a las siguientes: 
1. El arca de los pensamientos.

7. Competencia Intelectual y Tecnología de la Información (CITI).

16. Habitúate [Get used to].

22. Lucas y el caso del cuadro robado.

28. Neighborhood mapmachine.

35. Pictogram room.

Entre ellas encontramos una alta especialización en el caso de la aplicación 35, dado que trabaja dos áreas, mientras que el resto lo hacen entre tres y cinco.

\section{Discusión}

La incorporación de las aplicaciones informáticas a la mejora de la formación de los jóvenes y adultos con discapacidad intelectual queda muy lejos de la normalización didáctica (Casado-Muñoz, 2002) por distintas razones:

1. La primera: la cantidad. Como podemos observar, mientras que las propuestas para los jóvenes y adultos sin discapacidad aumentan de forma exponencial en los distintos niveles educativos, para nuestro colectivo el número es muy limitado. Específicamente en español sólo podemos indicar seis.

2. La segunda: la especificidad. Este tipo de aplicaciones, en muchas ocasiones, se diseñan para niños y, posteriormente, se señala en las indicaciones de uso que pueden ser utilizadas por jóvenes y adultos. Esta propuesta contribuye a mantener una infantilización de los usuarios, actitud rechazada reiteradamente (Robey, Beckley y Kirschner, 2006).

Para los jóvenes y adultos sin discapacidad, las distintas editoriales que diseñan y difunden recursos didácticos en los diferentes niveles educativos construyen portales, aplicaciones de acceso, contenidos específicos o de ampliación... para cada una de las materias implicadas en la formación. Así mismo, los profesores de las distintas materias desarrollan y comparten contenidos, apps, blogs, webs... que posteriormente pueden ser utilizados en otros centros y aulas. Esta realidad no la encontramos en el colectivo de jóvenes con discapacidad intelectual para quienes, ni por la cantidad, ni por las áreas de apoyo, se elaboran materiales adaptados, contradiciendo de esta forma la demanda del uso de las tecnologías en las mismas condiciones que sus pares sin discapacidad (Cullen y Albert, 2015). Las aplicaciones que encontramos están desarrolladas por fundaciones o investigadores. Aunque no hemos encontrado ninguna aplicación realizada específicamente por profesores o educadores para este colectivo, sí que podemos observar el uso con jóvenes y adultos de aplicaciones diseñadas para niños, por ejemplo, para trabajar conceptos básicos de lectura, de cálculo...

Las editoriales especializadas no dan respuesta al colectivo. En todo caso, como se ha dicho anteriormente, indican -creemos que erróneamente- que las aplicaciones pensadas y diseñadas para niños podrían ser directamente utilizables por jóvenes y adultos. Haciendo un análisis comparado con los otros colectivos sin discapacidad, 
pensemos en qué sucedería si a jóvenes de 15 o 16 años sin discapacidad se les propusiera que utilizaran materiales diseñados para niños de 8 o 9 años, aunque los contenidos abordados fueran coherentes con la edad. ¿Qué dirían los jóvenes?, ¿qué opinarían los padres y las madres?, ¿propondría estas actividades el profesorado?

Las aplicaciones específicas para jóvenes y adultos siguen siendo muy generalistas, alejadas de las necesidades de este grupo de edad, lo cual supone un riesgo en las posibles intervenciones en las que sean utilizadas. Según indican Ramdoss et al. (2012), el éxito recaería más en la calidad de la presentación y en los conocimientos y experiencia de los desarrolladores, que en los softwares en sí mismos.

La brecha digital existente en este colectivo (Abbot et al., 2011 y OECD, 2001), asociada al acceso a los contenidos, lejos de reducirse aumenta cada vez a mayor velocidad. En el caso de jóvenes y adultos, esta diferencia aumenta aún más que en los niños en edad escolar, pues los contextos de interacción social virtual también son más numerosos y variados.

Estudiando concretamente la incidencia de cada una de las áreas de apoyo, nos encontramos con una presencia en todas las aplicaciones de 2 de las subáreas de "Desarrollo humano". Ello evidencia que el trabajo de las habilidades implicadas se puede considerar de carácter transversal en relación con el uso de las Tecnologías de la Información y la Comunicación. Es decir, se trabajarán con todas las aplicaciones, tanto en aquellas diseñadas para el colectivo como en otras de carácter más generalista.

Si utilizamos como referencia esta área de apoyo, en la que se trabajan las habilidades psicomotoras y el refuerzo de procesos cognitivos básicos como la atención, la percepción y/o la memoria..., quedaría justificada la inclusión de todos estos programas informáticos susceptibles de ser usados por jóvenes y adultos con discapacidad, en el momento de definir perfiles de necesidades de apoyo.

Savidis et al. (2007) encuentran resultados similares de apoyo y trabajo de las áreas "Enseñanza y educación” y "Conductual”. Ellos evalúan dos aplicaciones multimedia y dos juegos diseñados específicamente para su estudio. Coinciden con los resultados que nosotros hemos obtenido en estas áreas, confirmando que la formación y entrenamiento de materias correspondientes a etapas escolares en general, junto con temas conductuales, son los que han gozado hasta ahora de un mayor interés por parte de investigadores y desarrolladores de recursos multimedia.

Continuando con el análisis a través de las subáreas, se nos presenta una realidad diferente, es decir, tenemos muchas posibilidades de trabajar aspectos puntuales de cada una de las áreas, sin profundizar en la diversidad de competencias asociadas a cada una de ellas. "Enseñanza y educación” y el área "Conductual” se trabajan en gran medida en edades inferiores, por lo que, como venimos indicando, aumenta la probabilidad de encontrar aplicaciones para niños que, por intereses comerciales, se propongan para jóvenes y adultos, aunque no se haya realizado ningún tipo de adaptación a las características psicosociales de estos últimos destinatarios.

El resto de las áreas cuentan con una presencia muy inferior en el conjunto analizado: en todos los casos, menor al 20\%. Respecto a las áreas con una tasa de presencia entre el 10 y el $20 \%$ de las aplicaciones ("Social”, "Vida en la comunidad" y "Salud y seguridad"), debemos indicar que: 
- Ciertas subáreas sí cuentan con algún tipo de apoyo que emplea dispositivos móviles, como, por ejemplo, uso de transportes públicos y elección de itinerarios (Mechling y O’Brien, 2010) o realización de compras (Ayres et al., 2006; Hansen y Morgan, 2008), si bien estos recursos no son aplicaciones multimedia e interactivas y se limitan a audios, imágenes o sistemas de instrucción solo por vídeo.

- Se han encontrado otras subáreas infratratadas en general, con tasas muy bajas, como participación social, servicios de emergencias: ambulancias, policía, bomberos..., o educación vial, a pesar de su alto interés para el desarrollo de habilidades de autonomía en la comunidad, como indican Wells et al. (2014).

Mucho más discreta es la presencia de apoyos en estas aplicaciones para el resto de áreas, destacando las de "Vida en el hogar", "Empleo" y "Protección y defensa" como aquellas menos tratadas. Aunque puntualmente encontramos 10 aplicaciones que abordan específicamente destrezas de compras relacionadas con el hogar (productos de limpieza, comida, etc.), 6 programas dedicados a entrenar destrezas de organización del espacio personal y 4 que trabajan las tareas básicas de limpieza. Se ha detectado poca o ninguna presencia de aplicaciones multimedia que trabajen algunas subáreas clave de las dos primeras, aunque sí encontramos evidencias de algunos apoyos tecnológicos que emplean sencillos audios, vídeos o Power Points no interactivos, para entrenar en la preparación de comidas (Ayres y Cihak, 2010 o Mechling, 2008) o para la preparación del currículum (Elleven, Wircenski, Wircenski y Nimon, 2006).

La situación más extrema es el caso de "Protección y defensa", área que, en general, no cuenta con ningún apoyo concreto en forma de aplicación multimedia ni tampoco en otros formatos (VW, instrucciones mediante vídeo, Power Points, etc.), a pesar de que es un área fundamental para llevar una vida independiente.

\section{Conclusiones}

El incremento de la brecha digital para las personas con discapacidad intelectual es una realidad perfectamente constatada por múltiples autores, pero esta realidad se encuentra mucho más marcada aún en el caso de los jóvenes y adultos.

La relación entre las aplicaciones encontradas con la mejora de las áreas de apoyo es muy desigual, encontrando áreas ampliamente abordadas y otras con presencia residual. Por ello, es necesario difundir el conocimiento de las áreas de apoyo entre los desarrolladores informáticos, de manera que permita un abordaje más amplio de las mismas. Es preciso también valorar que el área "Desarrollo humano" no se debe considerar un indicador importante para trabajar con estas herramientas, dado que con las TIC aparece como un área de carácter transversal y mínimamente discriminadora del valor de las aplicaciones.

Dado el escaso desarrollo de aplicaciones para el colectivo, particularmente llamativo en lengua española, coincidimos con Mechling y O'Brien (2010) al considerar que se precisa construir productos tecnológicos específicos que se adapten, realmente, 
APLICACIONES INFORMÁTICAS DIRIGIDAS A JÓVENES Y ADULTOS CON DISCAPACIDAD INTELECTUAL PARA EL DESARROLLO DE LAS ÁREAS DE APOYO

M. E. BAÑOS-GARCÍA, F. LEZCANO-BARBERO, E. GARCÍA-MATÉ Y R. CASADO-MUÑOZ

a las necesidades de jóvenes y adultos con DI, dado que los productos generalistas pueden tener interés sólo para algunas de las áreas de apoyo.

Esta situación nos enseña igualmente que es especialmente necesaria la formación en tecnología de los profesionales que trabajan con el colectivo, para facilitar así la construcción, difusión y uso de materiales adecuados.

\section{Referencias bibliográficas}

AAIDD (2010). Intellectual Disability: Definition, Classification and Systems of Support. Washington: AAIDD.

AAIDD (2012). User's Guide to Intellectual Disability: Definition, classification and Systems of Support. Washington: AAIDD.

Аввотт, С. (2007). Report 15, E-inclusion: Learning Difficulties and Digital Technologies Futurelab. Bristol (UK).

Аввоtт, C., Brown, D., Evett, L., Standen, P. y Wright, J. (2011). Learning difference and digital technologies: a literature review of research involving children and young people using assistive technologies 2007-2010. Disponible en http://bit.ly/29PwW7N.

Aguilar-Tamayo, M. F. (2004). El concepto de desarrollo en Vygotski como marco de reflexión para el uso de tecnologías en la enseñanza y aprendizaje en personas con discapacidad. Plasticidad y Restauración Neurológica, 3 (1 y 2), 45-58.

Ayres, K. y Cihak, D. (2010). Computer- and Video-Based Instruction of Food-Preparation Skills: Acquisition, Generalization, and Maintenance. Intellectual and Developmental Disabilities, 48 (3), 195-208. doi:http://dx.doi.org/10.1352/1944-7558-48.3.195.

Ayres, K. M., Langone, J., Boon, R. T. y Norman, A. (2006). Computer-based Instruction for Purchasing Skills. Education and Training in Developmental Disabilities, 41 (3), 253-263.

Buedo García, J. A. y Molina Moreno, V. (2007). Desarrollo de las TICs en los Discapacitados de Castilla-La Mancha. Colombia Digital. Descargado de www.colombiadigital.net website: http://www.elearningamericalatina.com/edicion/julio1_2007/tr_2.pdf.

Bull, G. (2009). Tutor, tool, tutee: A vision revisited. Contemporary issues in Technology and Teacher Education, 9 (2), 89-94.

Casado-Muñoz, R. (2002). Para continuar trabajando. Revista de Educación Especial, 31, 117-126.

Casado-Muñoz, R., Lezcano Barbero, F. y Rodríguez-Conde, M. (2015). Active Ageing and Access to Technology: An Evolving Empirical Study. Comunicar: Revista Científica de Comunicación y Educación, 45, 37-46. doi: https://doi.org/10.3916/C45-2015-04.

Cullen, J. M. y Albert-Morgan, S. R. (2015). Technology Mediated Self-Prompting of Daily Living Skills for Adolescents and Adults with Disabilities: A Review of the Literature. Education and Training in Autism and Developmental Disabilities, 50 (1), 43-55.

Davies, D. K., Stock, S. E. y Wehmeyer, M. L. (2001). Enhancing Independent Internet Access for Individuals with Mental Retardation through Use of a Specialized Web Browser: A Pilot Study. Education and Training in Autism and Developmental Disabilities, 36 (1), 107-113.

Dekelver, J. y De Boeck, J. (2010). People with Intellectual Disabilities at Risk of e-Exclusion. Paper presented at the Conference Proceedings eYouth Conference (28-29, May). Antwerp (Belgium).

EDUCARED (2005). Contenidos y Metodologías existentes en la red: informe del grupo de trabajo para el III Congreso Internacional de EducaRed. Madrid: EDUCARED. 
APLICACIONES INFORMÁTICAS DIRIGIDAS A JÓVENES Y ADULTOS CON DISCAPACIDAD INTELECTUAL PARA EL DESARROLLO DE LAS ÁREAS DE APOYO

M. E. BAÑOS-GARCÍA, F. LEZCANO-BARBERO, E. GARCÍA-MATÉ Y R. CASADO-MUÑOZ

Elleven, R., Wircenski, M., Wircenski, J. y Nimon, K. (2006). Curriculum-Based Virtual Field Trips: Career Development Opportunities for Students with Disabilities. Journal for Vocational Special Needs Education, 28 (3), 4-11.

García-Valcárcel Muñoz-Repiso, A. (2009). Medios Informáticos. Descargado de http:// web.usal.es/ anagv/arti5.htm.

Gros Salvat, B. (2003). Nuevos medios para nuevas formas de aprendizaje: el uso de los videojuegos en la enseñanza. Barcelona: Universidad de Barcelona.

Gutiérrez-Recacha, P. y Martorell-Cafranga, A. (2011). Las personas con discapacidad intelectual ante las TIC. Comunicar, Revista Cientifica de Educomunicación, 36 (XVIII), 173-180. doi: 10.3916/C36-2011-03-09.

Hansen, D. L. y Morgan, R. L. (2008). Teaching Grocery Store Puchasing Skills to Students with Intellectual Disabilities Using a Computer-Based Instruction Program. Education and Training in Autism and Developmental Disabilities, 43 (4), 431-442.

Hersh, M. A. (2010). The Design and Evaluation of Assistive Technology Products and Devices Part I: Design. En J. H. STONE (Ed.), International Encyclopedia of Rehabilitation. Descargado de http://cirrie.buffalo.edu/encyclopedia/en/article/309/.

Hinostroza, E., Heep, P., Mellar, H., Preston, C. y Rehbein, L. (1997). ¿Diseño de software educativo o de software escolar? Revista Informática Educativa. Proyecto SIIE, 10 (1), 57-73.

Hutcherson, K., Langone, J., Ayres, K. y Clees, T. (2004). Computer Asisted Instruction to Teach Item Selection in Grocery Stores: An Assessment of Acquisition and Generalization. Journal of Special Education Technology, 19 (4), 33-42.

Leahy, D. y Dolan, D. (2010). Digital Literacy: A Vital Competence for 2010? En N. ReyNOldS y M. TurcsánYi-Szabó (Eds.), Key Competencies in the Knowledge Society. IFIP International Federation for Information Processing (pp. 210-221). Brisbane, Australia.

Maeda, Y., Tsjimura, E., Sigita, K., Ока, T. y Yokota, M. (2009). An adaptive User Interface for Universal Multimedia Access. Paper presented at the International Conference on Complex, Intelligent and Software Intensive Systems, cisis'2009, Fukuoka.

Marqués Graells, P. (2010). Recursos de Tecnología Educativa. Descargado de http://www. xtec.cat/ pmarques/tecnoedu.htm.

Mechling, L. C. (2008). High Tech Cooking: A Literature Review of Evolving Technologies for Teaching a Functional Skill. Education and Training in Developmental Disabilities, 43 (4), 474-485.

Mechling, L. C. y O’Brien, E. (2010). Computer-Based Video Instruction to Teach Students with Intellectual Disabilities to Use Public Bus Transport. Education and Training in Autism and Developmental Disabilities, 45 (2), 230-241.

OECD (2001). Understanding the Digital Divide. Descargado de http://bit.ly/2a3kvsf.

Pablo-Blanco, C. De y Rodríguez, M. J. (2010). Manual práctico de discapacidad intelectual. Madrid: Síntesis, S. A.

Ramdoss, S., Lang, R., Fragale, C., Britt, C., O’Reilly, M., Sigafoos, J., Didden, R., PalMEN, A. y LANCIONI, G. E. (2012). Use of Computer-Based Interventions to Promote Daily Living Skills in Individuals with Intellectual Disabilities: A Systematic Review. Journal of Developmental and Physical Disabilities, 24, 197-215.

Robey, K. L., Beckley, L. y Kirschner, M. (2006). Implicit Infantilizing Attitudes About Disability. Journal of Developmental and Physical Disabilities, 18 (4), 441-453. doi: 10.1007/ s10882-006-9027-3.

SÁnchez Montoya, R. (2002). Ordenador y Discapacidad (2nd ed.). Madrid: Cepe S. L. 
Savidis, A., Grammenos, D. y Stephanidis, C. (2007). Developing inclusive e-learning and e-entertainment to effectively accommodate learning difficulties. Universal Accesss Information Society, 5, 401-419. doi: 10.1007/s10209-006-0059-3.

Schalock, R. L. y Verdugo, M. Á. (2003). Calidad de Vida. Manual para profesionales de la educación, salud y servicios sociales (M. Á. Verdugo y C. Jenaro, traducción). Madrid: Alianza Editorial S. A.

Stendal, K. (2012). How do People with Disability Use and Experience Virtual Worlds and ICT: A Literature Review. Journal of Virtual Worlds Research, 5 (1), 1-17.

SuÁrez Alonso, R. C. (2007). Tecnologías de la Información y la Comunicación. Introducción a los Sistemas de Información y de Telecomunicación. Vigo: Ideaspropias Editorial.

Verdugo, M. Á. y Gutiérrez Bermejo, B. (2009). Discapacidad intelectual. Adaptación social $y$ problemas de comportamiento (1. ${ }^{\mathrm{a}}$ ed.). Madrid: Ediciones Pirámide.

Verdugo, M. Á. y Schalock, R. L. (2010). Últimos avances en el enfoque y concepción de las personas con discapacidad intelectual. Siglo Cero, 41 (4), 7-21.

Wells, J., Clark, K. y Sarno, K. (2014). An Interactive Multimedia Program to Prevent Hiv Transmission in Men With Intellectual Disability. American Journal on Intellectual and Developmental Disabilities, 119 (3), 276-286. doi: 10.1352/1944-7558-119.3.276. 